\title{
Pulmonary intestinal adenocarcinoma secondary to intestinal metaplasia of bladder: a rare case report
}

\author{
Ao Li", Yubo Yang", Ping Han \\ Department of Urology/Institute of Urology, West China Hospital, Sichuan University, Chengdu, China \\ \#These authors contributed equally to this work. \\ Correspondence to: Ping Han. Department of Urology/Institute of Urology, West China Hospital, Sichuan University, Guoxue Xiang \#37, Chengdu \\ 610041, China. Email: hanpingwch@163.com.
}

\begin{abstract}
Intestinal metaplasia (IM) in the esophagus is considered as a risk factor of subsequent intestinaltype adenocarcinoma based on the classical theory of metaplasia-dysplasia-carcinoma. IM rarely occurs in urinary tract and fewer studies have been reported with long-term follow-up in those patients with IM of bladder. Thus, the etiology, pathological characteristics and prognosis of IM of urinary tract remain unclear. In the past, IM was regarded as benign lesions in bladder and there might have no relationship between IM of bladder and potential malignancy. Recently, some studies suggested that IM of bladder might indicate a risk of progression to carcinoma and require active surveillance according to their findings. However, due to limited cases, there has not yet been any systematic review or consensus on the management of IM of bladder. Here we reported a patient diagnosed as pulmonary intestinal adenocarcinoma suspiciously secondary to IM of bladder, without primary gastrointestinal cancer or bladder adenocarcinoma. Receiving lung surgery and adjuvant chemotherapy, the patient had no recurrence after a follow-up of 27 months. Hence, the present case demonstrated that ectopic IM can be a driving factor of adenocarcinoma in other organs, such as pulmonary intestinal adenocarcinoma, and it requires a long-term follow-up with overall examinations.
\end{abstract}

Keywords: Intestinal metaplasia (IM); bladder tumor; pulmonary intestinal adenocarcinoma; case report

Submitted Sep 10, 2020. Accepted for publication Feb 19, 2021.

doi: 10.21037/apm-20-1818

View this article at: http://dx.doi.org/10.21037/apm-20-1818

\section{Introduction}

Intestinal metaplasia (IM), which is considered as a risk factor of intestinal-type adenocarcinoma in esophagus on the basis of metaplasia-dysplasia-carcinoma progression, rarely occurs in bladder $(1,2)$. Although primary bladder adenocarcinoma only accounted for $0.5-2 \%$ of all patients diagnosed as bladder cancer, it usually resulted in worse prognosis due to high rate of recurrence and progression (3). However, the association between IM of bladder and risk of progression remained unclear. The etiology of IM of urinary tract is unknown. In the past decades, IM were regarded as a benign disease and had no relationship with bladder cancer, although it could be found adjacent to urothelial carcinoma, adenocarcinoma, or squamous cell carcinoma $(3,4)$. But in 2021, Acosta et al. (5) proclaimed that IM of bladder might indicate a risk of progression to carcinoma when it was found with highgrade dysplasia.

To our acknowledgement, there was no literature discussing any case of ectopic intestinal adenocarcinoma secondary to IM of bladder. We present the following rare case to demonstrate that IM of bladder should be paid attention and systematically surveilled because it might be recurrent or even progressive in other organs. We present the following case in accordance with the CARE reporting checklist (available at http://dx.doi.org/10.21037/apm-201818). 

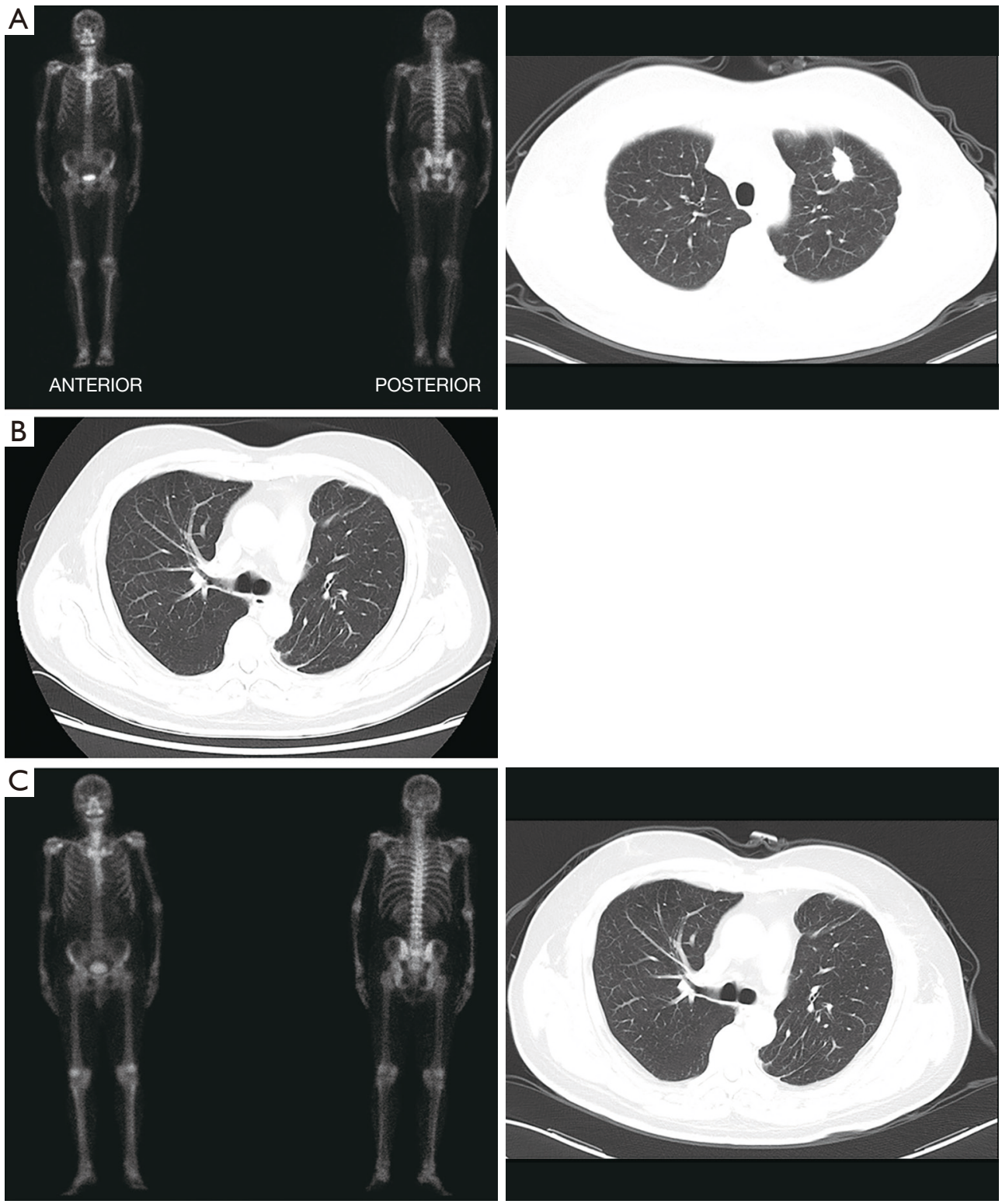

Figure 1 The CT images throughout the period: (A) Before surgery: the CT showed a neoplasm located in left upper lobe without metastasis of bone; (B) after complete chemotherapy course: the lesions were not found in chest CT; (C) there are no recurrence in recent review.

\section{Case presentation}

A 61-year-old male, complaining of hematuria for 5 days, was admitted to our center in Feb 2015. The ultrasound examination showed a tumor-like lesion in the bladder. The patient underwent a diagnostic transurethral resection of bladder tumor (TURBT), and a tumor was found on the right side of the bladder with $2 \mathrm{~cm} \times 2.5 \mathrm{~cm}$ in size. It was diagnosed as IM with high-grade intraepithelial dysplasia, due to the immunohistochemistry (IHC): CK7 (most -), CK20 (+), CDX2 (+), Villin (+), P63 (-), GATA-3 $(-)$, Uroplakin III (-), Ki67 (+, 30-40\%). The patient did not receive any medications except regular examination, no recurrence was observed in bladder. In Jun 2017, a neoplasm in his left lung was found by CT, but the bone scan did not indicate any metastasis (Figure 1A). Then, his upper lobe of left lung was resected with a video-assisted thoracic surgery 


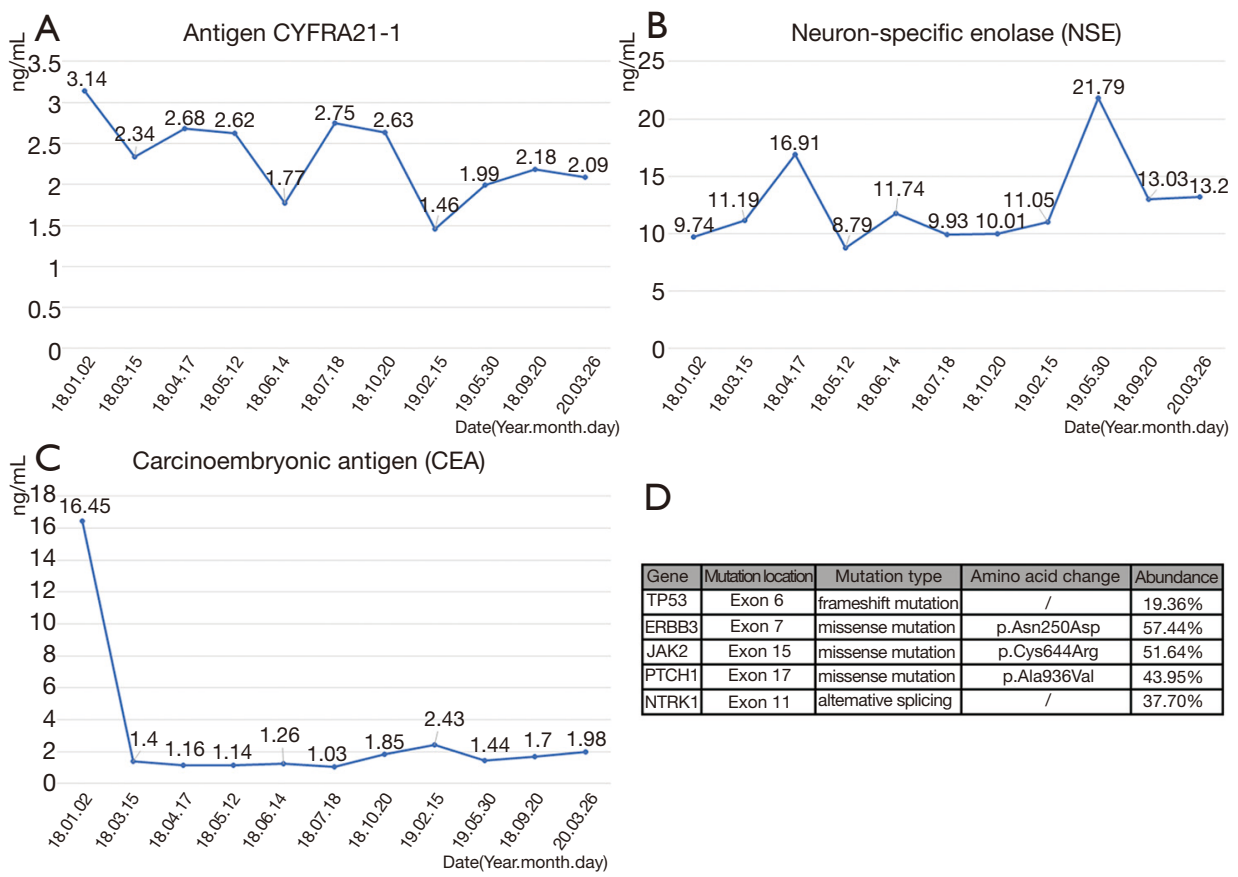

Figure 2 Serum levels of biomarkers and genetic assay. (A) Serum levels of CYFRA 21-1 (normal value: $<3 \mathrm{ng} / \mathrm{mL}$ ). (B) Serum levels of NSE (normal value: $<15 \mathrm{ng} / \mathrm{mL}$ ). (C) Serum levels of CEA (normal value: $<3.4 \mathrm{ng} / \mathrm{mL}$ ). (D) Genetic assay report.

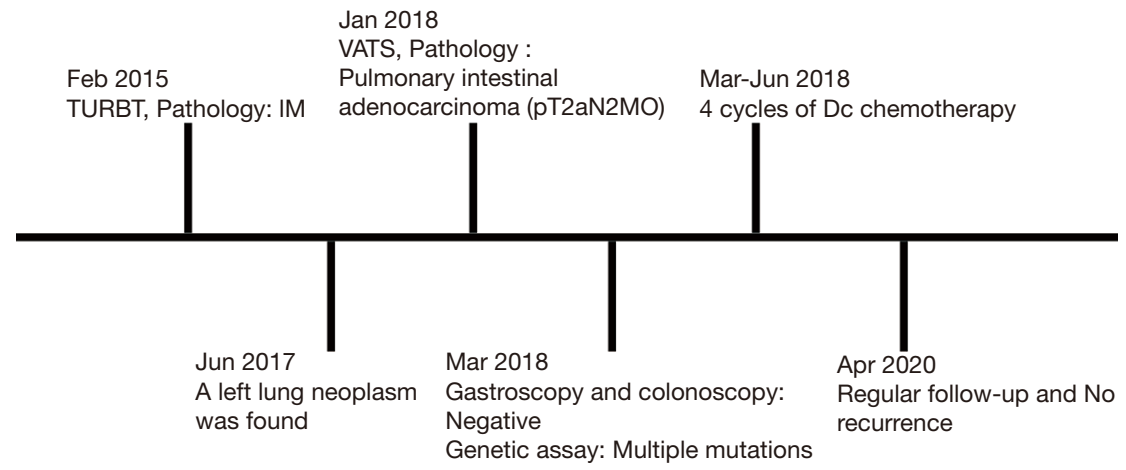

Figure 3 The timeline of the management.

(VATS). The IHC showed CK7 (-), CK20 (+), CDX-2 (+), Villin (+), EMA (+, weak), TTF-1 (+), NapsinA (-), MIB$1(+, \sim 90 \%)$, so it was diagnosed as pulmonary intestinal adenocarcinoma with lymph nodes invasion (pT2aN2M0). Then, he underwent a gastroscopy and colonoscopy, but no neoplasm was detected From March to June 2018, the patient received four cycles of chemotherapy of Doxorubicin plus Cyclophosphamide. Till now no recurrence had been found with a follow-up of 27 months (Figure 1B,C). Besides, the serum levels of carcinoembryonic antigen (CEA), neuron-specific enolase (NSE) and cytokeratin 19 fragment
(CYFRA 21-1) were continuously monitored, CEA dropped significantly and had kept at a low level since the lung surgery was performed (Figure $2 A, B, C$ ). Meanwhile, the genetic assay of lung specimen showed multiple mutations, especially the frameshift mutation of TP53 exon6 and missense mutation of ERBB3 exon7 (Figure 2D). The timeline of the management was presented in Figure 3.

All procedures performed in studies involving human participants were in accordance with the ethical standards of the institutional and/or national research committee(s) and with the Helsinki Declaration (as revised in 2013). Written 
informed consent was obtained from the patient.

\section{Discussion}

In the present case, the pulmonary intestinal adenocarcinoma was mostly secondary to the IM of bladder for the IHC results were extremely similar. The pulmonary adenocarcinoma probably originated from primary lesions of bladder, but it was more likely another manifestation of IM related mutation or "ectopic intestinal neoplasm syndrome". This case provided an evidence that IM of bladder should be regarded as a precursor of adenocarcinoma, in accordance with Acosta et al. (5) finding. Additionally, we detected dysplasia in the present case, which may account for the secondary pulmonary adenocarcinoma without any evidence of primary lung adenocarcinoma. Although IM was not malignancy in the view of pathology, it triggered the signal of caution that the patient might suffer intestinal adenocarcinoma in the next few years. In a study of 89 patients with IM of bladder, recurrence was detected in four patients and one developed bladder adenocarcinoma at six-month follow-up after TURBT (6).

It should be noticed that the mutations of TP53, ERBB3 and other oncogenes were detected in the present case (Figure 2D). Since the diagnostic model (based on FGFR3, TP53, PIK3CA, ARID1A, STAG2, and KTM2D gene mutation) and prognosis-predicting model (based on FGFR3, TP53, PIK3CA, and ERBB3 gene mutation) showed a satisfied capability in non-muscle-invasive bladder cancer (NMIBC) (7). TP53 and ERBB3 mutation in this case might demonstrate that IM represented the potential of invasion, and it was associated with carcinogenesis and recurrence of adenocarcinoma. JAK2 was involved in JAK2/ STAT pathway and associated with intrinsic apoptosis colon adenocarcinoma (8). PTCH1 is a tumor suppressor gene and the mutation of PTCH1 was found in intestinal ampullary cancer (9). As an oncogene, NTRK1 mutation could be detected in numerous adenocarcinomas and it might become a novel target of oncotherapy (10).

In some cases, tumor biomarkers could indicate cancer recurrence and progression. CYFRA 21-1 was proved to be exclusively expressed in lung tissues and regarded as the most sensitive marker for non-small-cell lung carcinoma (NSCLC), especially squamous carcinoma. Numerous multivariate analyses showed that CYFRA 21-1 was an important biomarker in predicting prognosis and response to therapy (11). NSE is a glycolytic enolase that is mainly found in the central nerve system, neuro- endocrinal tissues and peripheral neurons, with an satisfied sensitivity and specificity for the diagnosis and surveillance of small cell lung cancer(SCLC) and also of clinical value in NSCLC (12). In the present case, low levels of CYFRA 21-1 and NSE throughout also supported the fact that the pulmonary lesion was probably neither NSCLC nor SCLC (Figure 2A,B). As a biomarker for variety of adenocarcinoma, a significantly decreased level of CEA after surgery, as well as during the follow-up, indicated a satisfied efficacy of surgery and adjuvant chemotherapy.

Due to the extremely low incidence of IM of bladder, there have not been any guidelines or consensus about the management yet. It is apparent that our study is a case report with a low level of evidence. More systematic researches are needed to evaluate the pathological characteristics and appropriate clinical management of IM. Although IM can be associated with a concurrent diagnosis of carcinoma, no sufficient evidence suggested that it increases the future risk of malignancy, and surveillance cystoscopy in such patients were not recommended (3). On the contrary, frequent surveillance was necessary, particularly for those specimens with dysplastic changes (7). And we also strongly suggest that patients with IM of bladder should be active surveilled including a regular $\mathrm{CT}$ of chest and abdomen at a frequency of 6 to 12 months.

\section{Conclusions}

In summary, IM of the bladder, might be potentially associated with pulmonary secondary intestinal adenocarcinoma. A thorough examination should be performed once diagnosed as IM by TURBT. Besides, active surveillance including cancer biomarkers, CT of lung and abdomen, should also be regularly performed.

\section{Acknowledgments}

Funding: The study was supported by the 1.3 .5 project for disciplines of excellence, West China Hospital, Sichuan University (ZY2016104) and Pillar Program from Department of Science and Technology of Sichuan Province (2018SZ0219).

\section{Footnote}

Reporting Checklist: The authors have completed the CARE reporting checklist. Available at http://dx.doi.org/10.21037/ apm-20-1818 
Conflicts of Interest: All authors have completed the ICMJE uniform disclosure form (available at http://dx.doi. org/10.21037/apm-20-1818). The authors have no conflicts of interest to declare.

Ethical Statement: The authors are accountable for all aspects of the work in ensuring that questions related to the accuracy or integrity of any part of the work are appropriately investigated and resolved. All procedures performed in studies involving human participants were in accordance with the ethical standards of the institutional and/or national research committee(s) and with the Helsinki Declaration (as revised in 2013). Written informed consent was obtained from the patient.

Open Access Statement: This is an Open Access article distributed in accordance with the Creative Commons Attribution-NonCommercial-NoDerivs 4.0 International License (CC BY-NC-ND 4.0), which permits the noncommercial replication and distribution of the article with the strict proviso that no changes or edits are made and the original work is properly cited (including links to both the formal publication through the relevant DOI and the license). See: https://creativecommons.org/licenses/by-nc-nd/4.0/.

\section{References}

1. Young RH. Tumor-like lesions of the urinary bladder. Mod Pathol 2009;22 Suppl 2:S37-52.

2. DeMeester SR, DeMeester TR. Columnar mucosa and intestinal metaplasia of the esophagus: fifty years of controversy. Ann Surg 2000;231:303-21.

3. Zhong M, Gersbach E, Rohan SM, et al. Primary adenocarcinoma of the urinary bladder: differential

Cite this article as: Li A, Yang Y, Han P. Pulmonary intestinal adenocarcinoma secondary to intestinal metaplasia of bladder: a rare case report. Ann Palliat Med 2021;10(7):8391-8395. doi: 10.21037/apm-20-1818 diagnosis and clinical relevance. Arch Pathol Lab Med 2013;137:371-81.

4. Smith AK, Hansel DE, Jones JS, et al. Role of cystitis cystica et glandularis and intestinal metaplasia in development of bladder carcinoma. Urology 2008;71:915-8.

5. Acosta AM, Sholl LM, Fanelli GN, et al. Intestinal metaplasia of the urinary tract harbors potentially oncogenic genetic variants. Mod Pathol 2021;34:457-68.

6. Gordetsky J, Epstein JI. Intestinal metaplasia of the bladder with dysplasia: a risk factor for carcinoma? Histopathology 2015;67:325-30.

7. Xin Z, Zhao C, Huang T, et al. Intestinal metaplasia of the bladder in 89 patients: a study with emphasis on long-term outcome. BMC Urol 2016;16:24.

8. Ren S, Xing Y, Wang C, et al. Fraxetin inhibits the growth of colon adenocarcinoma cells via the Janus kinase 2/ signal transducer and activator of transcription 3 signalling pathway. Int J Biochem Cell Biol 2020;125:105777.

9. Kumari N, Singh RK, Mishra SK, et al. Prevalence and spectrum of pathogenic germline variants in intestinal and pancreatobiliary type of ampullary cancer. Pathol Res Pract 2020;217:153309.

10. Ouali K, Pellat A, Cohen R, et al. [NTRK Fusions: A new way of treatment for gastro-intestinal tumor?]. Bull Cancer 2020;107:447-57.

11. Zhu F, Zhang Y, Shi L, et al. Gene mutation detection of urinary sediment cells for NMIBC early diagnose and prediction of NMIBC relapse after surgery. Medicine (Baltimore) 2019;98:e16451.

12. Yang G, Xiao Z, Tang C, et al. Recent advances in biosensor for detection of lung cancer biomarkers. Biosens Bioelectron 2019;141:111416. 